\title{
ESTILO DE VIDA, ESTADO DE SALUD Y ANSIEDAD EN ESTUDIANTES DE LA UNIVERSIDAD DE HUELVA
}

\author{
Concha Martínez-García \\ Dpto. de Psicología Social, Evolutiva y de la Educación, \\ Universidad de Huelva (UHU), España. \\ concha.martinez@dpsi.uhu.es \\ Ana Merchán-Clavellino \\ Dpto. de Psicología, Universidad de Cádiz (UCA), España. \\ Instituto Universitario de Investigación para el Desarrollo Social Sostenible (INDESS), \\ Yolanda Medina-Mesa \\ Centro de Psicología Yolanda Mesa, Cádiz. España. \\ Rocío Guil \\ Dpto. de Psicología, Universidad de Cádiz (UCA), España. \\ Instituto Universitario de Investigación para el Desarrollo Social Sostenible (INDESS),
}

Fecha de Recepción: 10 Febrero 2018

Fecha de Admisión: 10 Abril 2018

\section{RESUMEN}

Los informes de la OMS sobre la salud de jóvenes de 20 a 24 años, indican numerosos factores de riesgos sanitarios que aumentan las cargas de morbilidad, siendo además esta actual cohorte de jóvenes la mayor que ha existido jamás en el mundo. Entre estos factores, se citan los trastornos psicopatológicos de ansiedad y depresión, alteraciones alimentarias, consumo de alcohol y otras drogas ilícitas y hábitos comportamentales de riesgo. Este estudio tiene como objetivo describir algunos aspectos del estilo de vida, estado de salud autopercibido y ansiedad en una muestra de jóvenes estudiantes sanos de la Universidad de Huelva. Los instrumentos utilizados fueron la entrevista semiestructurada sobre el estilo de vida y estado de salud CRD (Martínez-García, et al., 2014) y el ISRA (Miguel-Tobal y Cano-Vindel, 2002). Participaron 102 universitarios (80.4\% mujeres), con edad media de 21.71 años (DT= 4.167). Los resultados mostraron tener un nivel socioeconómico medio (82.4\%) y un estilo de vida en el entorno familiar, en general, saludable. Sobre salud, sin embargo, se destaca que el $14,9 \%$ reconoce "beber alcohol más de lo que quisiera", de los que el $73.4 \%$, lo sitúan entre 2 y 3 copas de más y, hasta 7 copas de más, el resto. El 11.8\% mostró consumo de drogas ilícitas y la misma proporción fueron las/los que bajaron su peso en un $10 \%$ en los últimos seis meses. Los resultados del ISRA mostraron puntuaciones diagnósticas de Ansiedad severa en el $42.6 \%$ de la muestra, el $21.8 \%$ con A. marcada y el $9.9 \%$ con 


\title{
ESTILO DE VIDA, ESTADO DE SALUD Y ANSIEDAD EN ESTUDIANTES DE LA UNIVERSIDAD DE HUELVA
}

A. extrema, correlacionando con la variable alcohol los factores del ISRA Total ( $\left.T_{b}=-.205 ; p=.017\right)$, de Evaluación $\left(\mathrm{T}_{\mathrm{b}}=-.219 ; p=.011\right)$ y de Vida Cotidiana $\left(\mathrm{T}_{\mathrm{b}}=-.228 ; p=.007\right)$. Concluimos con la necesidad de activar los protocolos de prevención indicados por la OMS sobre estos factores, en el medio universitario.

Palabras clave: factores de riesgo en universitarios; habitos desadaptativos; ansiedad; consumo de sustancias

\begin{abstract}
Lifestyle, health status and anxiety in students of University of Huelva.

The WHO reports, concerning the health of young people aged from 20 to 24, highlight numerous health risk factors that increase the burden of morbidity, representing this current cohort of young people the largest that has ever existed in the world. Among these factors are included psychopathological disorders such as anxiety and depression, dietary alterations, alcohol and other illicit drugs consumption, and behavioral risk habits. This study aims to describe some aspects of lifestyle, self-perceived health status, and anxiety in a sample of healthy young students from the University of Huelva. The instruments used were a semi-structured interview on lifestyle and health status CRD (Martínez-García, et al., 2014) and ISRA (Miguel-Tobal \& Cano-Vindel, 2002). Participants were 102 university students ( $80.4 \%$ women), with an average age of 21.71 years (SD $=4.167)$. The results showed that participants had, in general terms, average socioeconomic level $(82.4 \%)$ and healthy lifestyle in the family context. However, concerning their health status, it is noted that $14.9 \%$ of the sample admited "to drink more alcohol than they would like", of which $73.4 \%$, stated a consumption between 2 and 3 drinks and the remaining up to 7 drinks. The $11.8 \%$ of the sample showed illicit drugs consumptionand thesame proportion those who lost $10 \%$ of their weight in the last six months. The ISRA results showed diagnostic scores of severe Anxiety in $42.6 \%$ of the sample, $21.8 \%$ with marked anxiety, and $9.9 \%$ with extreme anxiety, correlating with alcohol variable the factors of the Total ISRA (Tb $=-.205 ; p=.017)$, Evaluation $(\mathrm{Tb}=-.219 ; \mathrm{p}=.011)$ and Daily Life $(\mathrm{Tb}=-.228 ; \mathrm{p}=.007)$. As a conclusion, we manifest the need to activate prevention protocols indicated by the WHO on these factors, in the university environment.
\end{abstract}

Keywords: risk factors in university students; maladaptive habits; anxiety; substance use

\section{INTRODUCCIÓN}

Al establecer cuantitativamente los principales determinantes de la salud de las personas, se muestran cuatro factores esenciales. El primero de ellos que debe considerarse por ser el de mayor peso de influencia (43\%) es, el estilo de vida individual, seguido por la herencia genética que representa el $27 \%$, el entorno 0 ambiente en el que se vive, con el $19 \%$ y, el $11 \%$ restante, supone la influencia que aporta el sistema sanitario (Cecilia, Atucha, \& García-Estañ, 2017).

El estilo de vida, a su vez, está condicionado e integrado por una conjunción de factores, comportamientos y hábitos, favorables y no favorables, entre los que se destacan la dieta, el tabaco, el alcohol, el consumo de sustancias de abuso, la actividad física, la conducta sexual, el estrés, depresión y ansiedad, siendo además mediados por los factores socioeconómicos (García-Laguna, García-Salamanca, Tapiero-Paipa, Ramos, \& Marcela, 2012; Prat, 2007).

Estos condicionantes del estado de salud son especialmente relevantes en población joven. La actual transición demográfica cada vez afecta a un número mayor de países, llegándose a constituir la mayor cohorte de jóvenes que haya existido jamás en el mundo (Asamblea Mundial OMS, 2011). Estos informes mundiales de la salud de jóvenes de 20 a 24 años, indican que dichos factores de riesgos sanitarios aumentan las cargas de morbilidad en este grupo etario y, también, el aumento 
de las tasas de mortalidad de forma pronunciada desde la adolescencia (10-14 años), hasta los inicios de la edad adulta (20-24 años).

En ese estrato etáreo, la gran mayoría de jóvenes se encuentra en pleno proceso formativo, bien sea hacia la vía profesionalizante, o inmersos en los actuales Grados universitarios, especialmente, si atendemos a los niveles lamentables de desempleo de nuestro país que apenas dejan lugar a una opción alternativa diferente a la de los estudios.

Es también fácil entrever que esta situación incrementa la influencia otro de los factores de riesgo para la salud mencionados, la ansiedad y el estrés (Martínez-Otero Pérez, 2014). La vivencia de un futuro laboral incierto y desesperanzador tras finalizar la formación universitaria, u otra, la competitividad antes y después de obtener la titulación, así como los cambios cotidianos y de hábitos que acontecen durante la formación, como la separación del entorno familiar, reeducación de ocupaciones y actividades ante las exigencias académicas, por citar algunos, son todos ellos aspectos que inciden en la aparición de ansiedad patológica en universitarios (Balanza, Morales-Moreno, \& Guerrero-Muñoz, 2009; Márquez, Montorio, Izal, \& Losada, 2006).

Para intentar reducir en lo posible los mencionados factores riesgos para la salud y, por ende, dichas consecuencias, la intervención propuesta supone la apuesta decisiva de Programas de promoción de la salud, de forma integral y desde todos los ámbitos e instituciones, tanto educativas, como sanitarias, de políticas gubernamentales y estamentos privados (OMS, 2002).

En universidades iberoamericanas sí ha estado instaurándose el concepto y práctica de Universidades Saludables indicados por la OMS (Bastías-Arriagada \& Stiepovich-Bertoni, 2014; Blanco, Torres, Salazar, \& Parra, 2017; Lema-Soto et al., 2009; Muñoz \& Cabieses, 2008; Páez, 2012), sin embargo, las universidades españolas centran la promoción del estilo de vida saludable sobre la formación teórico-práctica impartida en materias concretas de disciplinas relacionadas con la salud, no transversalmente, ni de forma integral, aplicando iniciativas centradas en el ejercicio físico como apenas el único promotor de hábitos saludables e investigando sobre algunos de estos factores de riesgo de forma aislada (Barrios-Vicedo et al., 2015; Fernández Villa et al., 2013; Jiménez, Martínez, Miró, \& Sánchez, 2008), siendo escasos los estudios sobre el estilo de vida y estado de salud que integran los principales factores de riesgo en universitarios españoles (Martín-Monzón, 2007).

Este estudio tiene como objetivo describir, en una muestra de jóvenes estudiantes sanos de la Universidad de Huelva, algunos aspectos implicados en el estilo de vida, estado de salud autopercibido y ansiedad, así como las correlaciones entre estas variables y con el sexo y edad.

\section{MÉTODO}

Se trata de un diseño no experimental, de corte transversal y descriptivo, cuya selección de la muestra consistió en un proceso de muestreo no probabilístico de conveniencia o intencional.

\section{Participantes}

La muestra del estudio la constituyeron 102 universitarios de ambos sexos matriculados en segundo curso de Grado de la Universidad de Huelva. Su inclusión en el estudio implicó la firma previa del consentimiento informado que establece la Ley Orgánica 15/1999 de Protección de Datos de Carácter Personal.

\section{Instrumentos}

Los instrumentos utilizados fueron la entrevista semiestructurada, heteroadministrada sobre el estilo de vida y estado de salud CRD (Martínez-García, 2014) y el ISRA (Miguel-Tobal \& Cano-Vindel, 2002). 
La entrevista CRD (Apéndice) recoge datos sociodemográficos, nivel socioeconómico e ítems relativos al estilo de vida (EV), que explora aspectos de hábitos de sueño, alimentación, ocio y tiempo libre, preocupación económica, proyección estética, sentimiento de pertenencia y de apoyo familiar, así como sensación de logros conseguidos. Los ítems sobre el estado de salud (ES) recogen los principales factores de riesgos identificados en población joven, como son consumos de tabaco, alcohol, drogas ilícitas, psicofarmacología, pérdida importante de peso en los últimos y problemas de salud y hormonales.

\section{RESULTADOS}

Los resultados sociodemográficos mostraron una mayor proporción de mujeres $80.4 \%$, y la edad media del total fue 21.71 años (DT= 4.167). Los referidos al nivel socioeconómico también resultaron en su mayoría con un nivel medio (82.4\%), de los que el $59 \%$, contaban con residencia familiar en propiedad y el resto con hipoteca.

La Tabla 1 muestra los resultados de respuesta a los ítems referidos al Estilo de Vida (EV). Al ser una entrevista heteroadministrada, las respuestas dicotómicas reflejaban las conclusiones de los participantes a las explicaciones semiabiertas a las que se referían.

Tabla 1

Resultados de frecuencia absoluta y relativa sobre los ítems del EV

\begin{tabular}{lrrr}
\hline Ítems ESTILO DE VIDA & Respuesta & $\begin{array}{r}\text { Frec. absolutas } \\
(\mathrm{n})\end{array}$ & $\begin{array}{r}\text { Frec. relativas } \\
(\%)\end{array}$ \\
\hline ¿Le preocupa no dormir lo necesario? & Sí & 70 & 68,63 \\
& No & 32 & 31,37 \\
¿Llega económicamente a final de mes & & & \\
sin problemas? & Sí & 84 & 82,35 \\
& No & 18 & 17,65 \\
En caso de ser mujer ¿se pinta los & & & \\
labios o los ojos antes de salir? & Sí & 78 & 76,40 \\
En caso de ser varón ¿se arregla & No & 4 & 4,00 \\
antes de salir? & Sí & 17 & 16,60 \\
& No & 3 & 3,0 \\
¿Su familia le considera & & & \\
imprescindible? & Sí & 95 & 93,14 \\
¿Estás a gusto con lo que haces o con & No & 7 & 6,86 \\
lo que tienes? & Sí & 93 & 91,18 \\
& No & 9 & 8,82 \\
¿Procura hacer tres comidas/día? & & & \\
& Sí & 73 & 71,57 \\
¿Come en casa habitualmente? & No & 29 & 28,43 \\
& Sí & 95 & 93,14 \\
¿Tiene tiempo para sus gustos? & No & 7 & 6,86 \\
& Sí & 84 & 82,35 \\
\hline$*$ Na cor & 18 & 17,65 \\
\hline
\end{tabular}

**La correlación es significativa al nivel 0,01 (bilateral).

*La correlación es significativa al nivel 0,05 (bilateral).

Del resumen de los resultados sobre el Estilo de Vida del CRD, podría concretarse a modo de conclusión un perfil del estilo de vida de esta muestra de jóvenes universitarios, indicando que sí les preocupa no dormir lo necesario, no perciben tener problemas económicos, cuidan de su aspec- 
to, tienen la percepción de ser importantes para su familia, disponen de tiempo para su ocio y gustos y tienen hábitos alimentarios saludables al procurar llevar a cabo la comida tradicional y en su entorno familiar.

Similarmente, la Tabla 2 muestra los resultados del CRD correspondientes a los ítems sobre el Estado de Salud (ES).

Tabla 2

Resultados de frecuencia absoluta y relativa sobre los ítems del ES

\begin{tabular}{lrrr}
\hline Ítems ESTADO DE SALUD & Respuesta & $\begin{array}{r}\text { Frec. absolutas } \\
(\mathrm{n})\end{array}$ & $\begin{array}{r}\text { Frec. relativas } \\
\text { (\%) }\end{array}$ \\
\hline ¿Fumas diariamente? & Sí & 25 & 24,51 \\
& No & 77 & 75,49 \\
¿Bebes alcohol más de lo que & Sí & 15 & $\underline{14,70}$ \\
quisieras? & No & 86 & 85,30 \\
& & & \\
¿Cuántas copas crees que bebes de & $\leq 3$ & 11 & $\underline{73,33}$ \\
más? & $>3$ & 4 & $\underline{26,66}$ \\
¿Consumes otras drogas? & Sí & 12 & 11,76 \\
& No & 90 & 88,24 \\
¿Estás tomando algún fármaco o haces & Sí & 3 & 2,94 \\
algún seguimiento psicofarmacológico & No & 99 & 97,06 \\
por problemas de SM.? & & & \\
& & 7 & 6,86 \\
¿Tienes problemas de hormonas del & Sí & 95 & 93,14 \\
tiroides, suprarrenales o de hipófisis? & No & & \\
& & 12 & 11,76 \\
¿Has perdido/ganado más del 10\% de & Sí & 90 & 88,24 \\
tu peso en los últimos 6 meses? & No & 28 & 27,45 \\
¿Sufres alguna enfermedad? & Sí & 74 & 72,55 \\
\hline & No & &
\end{tabular}

**La correlación es significativa al nivel 0,01 (bilateral).

*La correlación es significativa al nivel 0,05 (bilateral).

De los resultados sobre el Estdo de Salud, se destaca que el $24,51 \%$ de la muestra es fumador habitual y el 14,7\% reconoce "beber alcohol más de lo que quisiera", de los que el $73.4 \%$, lo sitúan entre 2 y 3 copas de más y, el resto, hasta 7 copas de más. El 11.8\% mostró consumo de drogas ilícitas y la misma proporción fueron las/los que bajaron su peso en un $10 \%$ en los últimos seis meses.

LLama la atención que el $27,45 \%$ de los jóvenes universitarios participantes indiquen sufrir alguna enfermedad, refiriendo estar relacionadas en su mayoría con problemas alimentarios, de tiroides y con procesos ansioso-depresivos.

Respecto al Inventario de Situaciones y Rasgos de Ansiedad, los resultados procedentes de las puntuaciones directas de los participantes en el ISRA, fueron tratados para su normalización a puntuaciones centiles (Tabla 3). La clasificación de resultados del ISRA Total, que darían lugar a las codificaciones diagnósticas de Ansiedad, se muestran en la Figura 1. 
Tabla 3

Descriptivos de puntuaciones centiles, medias, dispersión y significacción estadística por sexo de la muestra en las distintas subescalas del ISRA

\begin{tabular}{l|r|r|r|r|c}
\hline \multirow{2}{*}{ ISRA } & Sexo & Media & DT & $\mathrm{t}$ & \multicolumn{1}{c}{$p$} \\
\hline Cognitivo (p.centil) & $\mathrm{M}$ & 69,60 & 25,463 & 2,107 & $.038^{*}$ \\
& $\mathrm{H}$ & 55,95 & 27,714 & & \\
\hline Fisiológico (p.centil) & $\mathrm{M}$ & 74,03 & 23,547 & 2,093 & $.046^{*}$ \\
& $\mathrm{H}$ & 59,20 & 29,409 & & \\
\hline Motor (p.centil) & $\mathrm{M}$ & 67,95 & 25,996 & 4,538 & $.000^{* * *}$ \\
& $\mathrm{H}$ & 38,00 & 28,023 & & \\
\hline TOTAL (p.centil) & $\mathrm{M}$ & 3,41 & 1,076 & 2,384 & $.019^{*}$ \\
& $\mathrm{H}$ & 2,75 & 1,251 & & \\
\hline Factor Evaluación (p.centil) & $\mathrm{M}$ & 72,79 & 26,209 & 2,250 & $.027^{*}$ \\
& $\mathrm{H}$ & 57,65 & 29,653 & & \\
\hline Factor Interpersonal (p.centil) & $\mathrm{M}$ & 66,03 & 26,904 & 1,871 & .064 \\
& $\mathrm{H}$ & 53,20 & 29,448 & & \\
\hline Factor Fóbico (p.centil) & $\mathrm{M}$ & 67,10 & 25,781 & 2,145 & $.034^{*}$ \\
& $\mathrm{H}$ & 53,00 & 28,303 & & \\
\hline Factor vida cotidiana (p.centil) & $\mathrm{M}$ & 70,14 & 24,569 &, 817 & .416 \\
& $\mathrm{H}$ & 65,05 & 26,339 & & \\
\hline
\end{tabular}

$* * *$ La correlación es significativa al nivel 0,00 (bilateral).

**La correlación es significativa al nivel 0,01 (bilateral).

*La correlación es significativa al nivel 0,05 (bilateral).

Figura 1. Clasificaciones diagnósticas de las puntuaciones centiles obtenidas por la muestra en el ISRA.

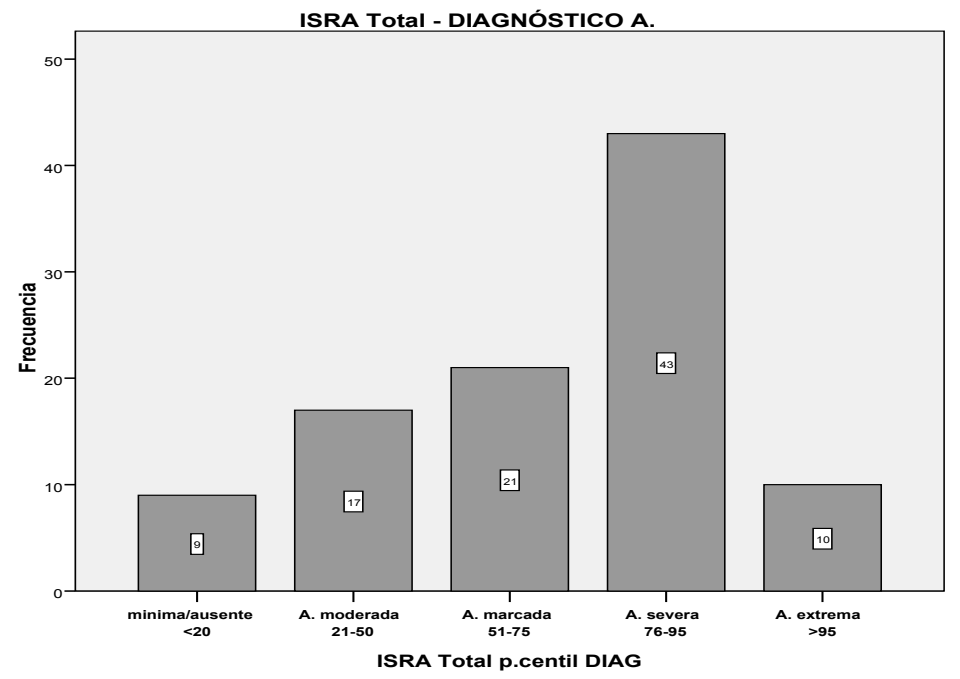

Los resultados del ISRA mostraron puntuaciones diagnósticas de Ansiedad severa en el $42.6 \%$ de la muestra, el $21.8 \%$ con A. marcada y el $9.9 \%$ con A. extrema.

Finalmente, con objeto de clarificar las siguientes Tablas 4-6, se exponen los resultados del análisis correlacional de las variables del estudio que han sido significativos. 
Tabla 4

Correlaciones significativas entre las variables sexo y edad con el Estilo de Vida, Estado de Salud y el ISRA.

\begin{tabular}{|c|c|c|c|c|c|c|c|}
\hline \multirow{2}{*}{\multicolumn{2}{|c|}{ Tau-b de Kendall }} & \multicolumn{2}{|c|}{ Estilo de vida } & \multirow{2}{*}{\begin{tabular}{l}
\multicolumn{1}{c}{$\begin{array}{c}\text { Estado de } \\
\text { Salud }\end{array}$} \\
¿Fuma \\
diariamente?
\end{tabular}} & \multicolumn{3}{|c|}{ ISRA } \\
\hline & & \multirow{2}{*}{$\begin{array}{l}\begin{array}{l}\text { Vive } \\
\text { con sus } \\
\text { padres }\end{array} \\
.271^{* *}\end{array}$} & $\begin{array}{l}\text { ¿Le } \\
\text { preocupa no } \\
\text { dormir lo } \\
\text { necesario? }\end{array}$ & & $\begin{array}{l}\text { ISRA } \\
\text { Total } \\
\text { (p. } \\
\text { centil) } \\
\end{array}$ & $\begin{array}{l}\text { Factor } \\
\text { Evaluación } \\
\text { (p. centil) }\end{array}$ & $\begin{array}{l}\text { Factor } \\
\text { Fóbico } \\
\text { (p. } \\
\text { centil) }\end{array}$ \\
\hline Edad & $\begin{array}{l}\text { Coeficiente } \\
\text { de } \\
\text { correlación }\end{array}$ & & & $-.235^{* *}$ & & & \\
\hline & Sig.(bilateral) & .002 & & .006 & & & \\
\hline & $\mathrm{N}$ & 102 & & 102 & & & \\
\hline \multirow[t]{3}{*}{ Sexo } & $\begin{array}{l}\text { Coeficiente } \\
\text { de } \\
\text { correlación }\end{array}$ & & $.198 *$ & & $-.199 *$ & $-.176^{*}$ & $-.171 *$ \\
\hline & $\begin{array}{l}\text { Sig. } \\
\text { (bilateral) }\end{array}$ & & .046 & & .020 & .039 & .043 \\
\hline & $\mathrm{N}$ & & 102 & & 101 & 101 & 101 \\
\hline
\end{tabular}

**La correlación es significativa al nivel 0,01 (bilateral).

*La correlación es significativa al nivel 0,05 (bilateral).

Las relaciones positivas entre la edad y vivir en la residencia familiar indican que a mayor edad de sujetos de la muestra, más tienen a vivir con los padres. La relación negativa de la edad con el hábito tabáquico muestran la relación inversa de que a medida que avaza la edad, se fuma menos (Tabla 4).

Las correlaciones entre el sexo y la preocupación por no dormir lo necesario, muestran que los hombres tienden a no darle importancia, mientras que a las mujeres sí les preocupa. Sobre las relaciones inversas de la variable sexo con el ISRA, supone la que mujeres puntuan con niveles de ansiedad más altos que los hombres (Tabla 4).

Tabla 5

Correlaciones significativas entre ítems del Estilo de Vida y Factores del ISRA.

\begin{tabular}{|c|c|c|c|c|c|}
\hline \multicolumn{2}{|c|}{ Tau-b de Kendall } & $\begin{array}{l}\text { ¿Está a } \\
\text { gusto con lo } \\
\text { que hace? }\end{array}$ & $\begin{array}{l}\text { ¿Procura } \\
\text { hacer las tres } \\
\text { comidas? }\end{array}$ & $\begin{array}{l}\text { ¿Come en casa } \\
\text { habitualmente? }\end{array}$ & $\begin{array}{l}\text { ¿Tiene } \\
\text { tiempo para } \\
\text { sus gustos? }\end{array}$ \\
\hline $\begin{array}{l}\text { ¿Llega a } \\
\text { final de } \\
\text { mes sin } \\
\text { problemas? }\end{array}$ & $\begin{array}{l}\text { Coeficiente } \\
\text { de } \\
\text { correlación } \\
\text { Sig.(bilateral) } \\
\text { N }\end{array}$ & $\begin{array}{c}.002 \\
102\end{array}$ & & & \\
\hline \multirow{4}{*}{$\begin{array}{l}\text { En caso } \\
\text { mujer, ¿se } \\
\text { pinta los } \\
\text { labios o los } \\
\text { ojos antes } \\
\text { de salir? }\end{array}$} & $\begin{array}{l}\text { Coeficiente } \\
\text { de } \\
\text { correlación }\end{array}$ & & $.228 *$ & $.257^{*}$ & $.349 * *$ \\
\hline & Sig.(bilateral) & & .041 & .021 & .002 \\
\hline & $\mathrm{N}$ & & 82 & 82 & 82 \\
\hline & & \multicolumn{2}{|c|}{ ISRA Factor impersonal } & \multicolumn{2}{|c|}{ ISRA Factor Fóbico } \\
\hline $\begin{array}{l}\text { ¿Le } \\
\text { preocupa } \\
\text { no dormir } \\
\text { lo } \\
\text { necesario? }\end{array}$ & $\begin{array}{l}\text { Coeficiente } \\
\text { de } \\
\text { correlación } \\
\text { Sig.(bilateral) } \\
\text { N }\end{array}$ & \multicolumn{2}{|c|}{$-.180^{*}$} & \multicolumn{2}{|c|}{$-.219 * *$} \\
\hline
\end{tabular}


La Tabla 5 resume las correlaciones significativas positivas entre los mismos ítems del Estilo de Vida y muestran relación directa entre llegar a final de mes sin apuros económicos y e s t a $r$ satisfecho con lo que hace. En el mismo sentido se relacionan que las mujeres que sí cuidan de su aspecto físico, tienen hábitos alimentarios más saludables y percepción de tener tiempo para sus hobbies.

También existe correlación entre la preocupación por no dormir lo necesario y con los factores de ISRA Impersonal y Factor Fóbico, indicando con los valores negativos o relaciones inversas, que cuanto menos importancia se dé al descanso, más elevadas son las puntuaciones en estos factores del ISRA (Tabla 5).

Tabla 6

Correlaciones significativas entre las variables Estado de Salud y del ISRA.

\begin{tabular}{|c|c|c|c|}
\hline \multirow[b]{2}{*}{$\begin{array}{l}\text { ISRA } \\
\text { Tau-b de Kendall }\end{array}$} & & \multicolumn{2}{|c|}{ Estado de Salud } \\
\hline & & $\begin{array}{l}\text { ¿Bebes alcohol más de } \\
\text { los que quisieras? }\end{array}$ & $\begin{array}{l}\text { ¿Estás tomando algún } \\
\text { fármaco o psicofármaco } \\
\text { por problemas de SM? }\end{array}$ \\
\hline \multirow[t]{3}{*}{ ISRA Total (p. centiles) } & $\begin{array}{l}\text { Coeficiente de } \\
\text { correlación }\end{array}$ & $-.205^{*}$ & \\
\hline & Sig.(bilateral) & .017 & \\
\hline & $\mathrm{N}$ & 100 & \\
\hline \multirow[t]{3}{*}{$\begin{array}{l}\text { Factor Evaluación (p. } \\
\text { centiles) }\end{array}$} & $\begin{array}{l}\text { Coeficiente de } \\
\text { correlación }\end{array}$ & $-.219^{*}$ & \\
\hline & Sig.(bilateral) & .011 & \\
\hline & $\mathrm{N}$ & 100 & \\
\hline \multirow[t]{3}{*}{ Factor Fóbico (p. centiles) } & $\begin{array}{l}\text { Coeficiente de } \\
\text { correlación }\end{array}$ & & $-.183 *$ \\
\hline & Sig.(bilateral) & & .031 \\
\hline & $\mathrm{N}$ & & 101 \\
\hline \multirow[t]{3}{*}{$\begin{array}{l}\text { Factor vida cotidiana } \\
\text { (p. centiles) }\end{array}$} & $\begin{array}{l}\text { Coeficiente de } \\
\text { correlación }\end{array}$ & $-.228 * *$ & \\
\hline & Sig.(bilateral) & .007 & \\
\hline & $\mathrm{N}$ & 100 & \\
\hline
\end{tabular}

Las relaciones negativas encontradas entre los ítems del ES, sobre el exceso de consumo de alcohol y las variables del ISRA, indican que los participantes que puntúan menos en Ansiedad Total, en Ansiedad ante la Evaluación y en Ansiedad en la vida cotidiana, tienden a consumir más cantidad de alcohol de lo que le gustaría. De la misma forma ocurre respecto a las relaciones entre estar tratándose con farmacología y tener Ansiedad Fóbica, es decir, cuanto más tratamiento farmacológico, menos ansiedad fóbica presentan.

\section{COMENTARIOS}

Los factores de riesgo para la salud de los jóvenes aquí analizados, han tenido como objetivo de investigación describir tres ejes relacionados: aspectos implicados en el concepto de Estilo de Vida (EV), autopercepción del Estado de Salud (ES) y el factor de riesgo quizás más ampliamente estudiado en psicopatología de población joven, la Ansiedad. En base a los tres focos estudiados, se realizan las siguientes consideraciones de interés sobre nuestros resultados.

Respecto al primero de ellos, EV, al utilizar una entrevista semiestructurada con formato de eteroadministración como el CRD, además de obtener los datos concluyentes con respuestas 
dicotómicas expuestos en la sección de Resultados, se obtuvo una información más ampliada y precisa de aquellas cuestiones a las que debían dar respuesta.

Esto nos permitió confirmar que dichas respuestas otorgadas se correspodían fidedignamente a su intención de respuesta y, no al azar, para aquellos casos en los en principio, no tenían una respuesta clara, como era usual respecto a las situaciones socioeconómicas, la representación del $10 \%$ de su peso corporal, o lo referido a padecer enfermedades, en las que incluirían las alergias y procesos caterrales, si no se les indicaba expresamente que esas eran afecciones menores.

Los resultados del EV aquí obtenidos han podido esclarecer un perfil aproximado del estudiante universitario medio: gran parte de la muestra se correspondió con un nivel socioeconómico sin apuros y un estilo de vida en el entorno familiar, en general, saludable. No hemos encontrado estudios con la aplicación del EV del CRD.

El segundo eje principal del presente estudio se refiere al Estado de Salud, también evaluado con el CRD. Es destacable indicar, además de lo ya expuesto, que el ítem relativo al alcohol, da por hecho su consumo habitual y lo que indaga es la percepción de pérdida de control sobre el mismo cuando se ingiere de más. Esto está recogido en la entrevista de forma intencional con objeto de facilitar la sinceridad al comunicar su consumo, dado que los participantes de la muestra, pese a garantizársele la confidencialidad a través del Consentimiento Informado, son conscientes de que los evaluadores somos profesores de esa misma universidad.

Los resultados mostraron en 101 participantes un consumo habitual, especialmente durante los fines de semana, ya que solamente una joven ofreció absoluta seguridad de no tomar alcohol nunca.

En otro estudio (Pineda-García, G. et al., 2017), que utilizó también el mismo ítem sobre alcohol del ES del CRD mostraron que, los estudiantes que se sobrepasaban en más de 3 copas, tenían mayor obsesión y búsqueda de la delgadez, siendo las mujeres las que duplicaban sus medias respecto a los hombres. Nuestros resultados no son equiparables al no relacionar el alcohol con las conductas de búsqueda de la delgadez, ni hemos obtenido correlaciones significativas entre las variables sexo y alcohol, pero son similares los promedios de excesos de consumo de alcohol aquí obtenidos.

El último factor de riesgo analizado, la ansiedad, ha resultado ser especialmente preocupante dado que se trata de una muestra no-clínica, sino de jóvenes que en principio serían controles en cualquier estudio comparativo y, sin embargo, el $74,3 \%$ ha puntuado en el ISRA por encima del corte diagnóstico.

Estos resultados son similares a los encontrados en la bibliografía al respecto (Balanza et al., 2009; Martínez-Otero, 2014), incluida la superioridad de las mujeres, respecto a los hombres en puntuaciones más elevadas de ansiedad.

Concluimos con la necesidad de activar en el medio universitario los protocolos de prevención indicados por la OMS sobre estos factores.

\section{REFERENCIAS}

Asamblea Mundial OMS. (2011). Los jóvenes y los riesgos sanitarios: informe de la Secretaría. 64ª . Asamblea Mundial de la Salud., A64/25 Punto 13.16.

Balanza, S., Morales-Moreno, I., \& Guerrero-Muñoz, J. (2009). Prevalencia de ansiedad y depresión en una población de estudiantes universitarios: factores académicos y sociofamiliares asociados. Clínica y Salud, 20(2), 177-187.

Barrios-Vicedo, R., Navarrete-Muñoz, E. M., García de la Hera, M., González-Palacios, S., ValeraGran, D., Checa-Sevilla, J. F., ... Vioque, J. (2015). Una menor adherencia a la dieta mediterránea se asocia a una peor salud auto-percibida en población universitaria. Nutrición Hospitalaria, $31(2)$. 
Bastías-Arriagada, E. M., \& Stiepovich-Bertoni, J. (2014). Una revisión de los estilos de vida de estudiantes universitarios Iberoamericanos. Ciencia y enfermería, 20(2), 93-101.

Blanco, D. M. L., Torres, L. V. A., Salazar, M. E. H., \& Parra, M. O. N. (2017). La relación entre Educación para la Salud, la Promoción de la Salud y la Prevención del Alcoholismo en estudiantes universitarios. Revista Didasc@lia: Didáctica y Educación. ISSN 2224-2643, 7(4), 143164.

Cecilia, M. J., Atucha, N. M., \& García-Estañ, J. (2017). Estilos de salud y hábitos saludables en estudiantes del Grado en Farmacia. Educación Médica. https://doi.org/10.1016/j.edumed.2017.07.008

Fernández, T., Alguacil, J., Ayán, C., Bueno, A., Cancela, J. M., Capelo, R., ... Llorca, J. (2013). Proyecto UNIHCOS: cohorte dinámica de estudiantes universitarios para el estudio del consumo de drogas y otras adicciones. Revista Española de Salud Pública, 87(6), 575-585.

García-Laguna, D. G., García-Salamanca, G. P., Tapiero-Paipa, Y. T., Ramos, C., \& Marcela, D. (2012). Determinantes de los estilos de vida y su implicación en la salud de jóvenes universitarios. Hacia la Promoción de la Salud, 17(2), 169-185.

Jiménez, M. G., Martínez, P., Miró, E., \& Sánchez, A. I. (2008). Bienestar psicológico y hábitos saludables:? están asociados a la práctica de ejercicio físico? International Journal of Clinical and Health Psychology, 8(1).

Lema-Soto, L. F., Salazar, I. C., Varela, M. T., Tamayo, J. A., Rubio, A., \& Botero, A. (2009). Comportamiento y salud de los jóvenes universitarios: satisfacción con el estilo de vida. Pensamiento psicológico, 5(12).

Márquez, M., Montorio, I., Izal, M., \& Losada, A. (2006). Predicción del nivel de Ansiedad a partir de la intensidad emocional y el afrontamiento cognitivo en situaciones amenazantes en personas jóvenes y mayores. Ansiedad y estrés, 12.

Martínez-García, C. (2014). Procesos cognitivos y emocionales en trastornos de la conducta alimentaria. Percepción de la Imagen Corporal y Toma de Decisiones en Mujeres Onubenses (PICTODEMO),

367. http://hdl.handle.net/10272/8916

Martínez-Otero Pérez, V. (2014). Ansiedad en estudiantes universitarios: estudio de una muestra de alumnos de la Facultad de Educación [Anxiety in university students: study of a sample of students of the Faculty of Education]. ENSAYOS. Revista de la Facultad de Educación de Albacete, 29(2), 63-78. http://dx.doi.org/10.18239/ensayos.v29i2.384

Martín-Monzón, I. (2007). Estrés académico en estudiantes universitarios. Apuntes de psicología, 25(1), 87-99.

Miguel-Tobal, J. J., \& Cano-Vindel, A. (2002). Inventario de situaciones y respuestas de ansiedad (ISRA): Manual./Inventory of Situations and Responses of Anxiety (ISRA). Manual (5 rev. ed.). Madrid: TEA.[Links].

Muñoz, M., \& Cabieses, B. (2008). Universidades y promoción de la salud:? cómo alcanzar el punto de encuentro? Revista panamericana de salud pública, 24(2), 139-146.

OMS. (2002). Informe sobre la salud en el mundo. Reducir los riesgos y promover una vida sana. Recuperado 9 de abril de 2018, a partir de http://www.who.int/whr/2002/en/whr02_es.pdf

Páez, M. L. (2012). Universidades saludables: Ios jóvenes y la salud. Archivos de Medicina (Col), 12(2).

Pineda-García, G., Martínez-García, C., Arámburo, V., González, A., Gómez-Peresmitré, G., \& Platas, S. (2017). Consumo de alcohol y búsqueda de la delgadez en universitarios de Baja California. XXV. Congreso Mexicano de Psicología, 802-804. 
Prat, A. (2007). El estilo de vida como determinante de salud. J. Rodes, JM Piqué \& A. Trilla, Libro de la salud del hospital Clinic de Barcelona y la fundación BBVA, 65.

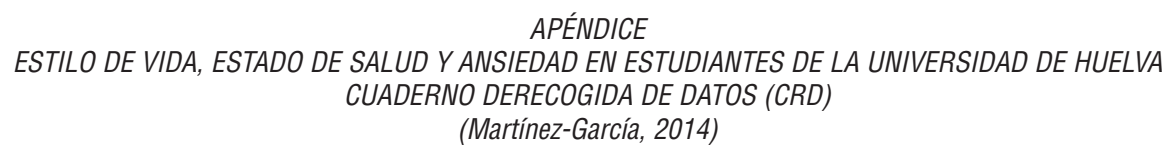

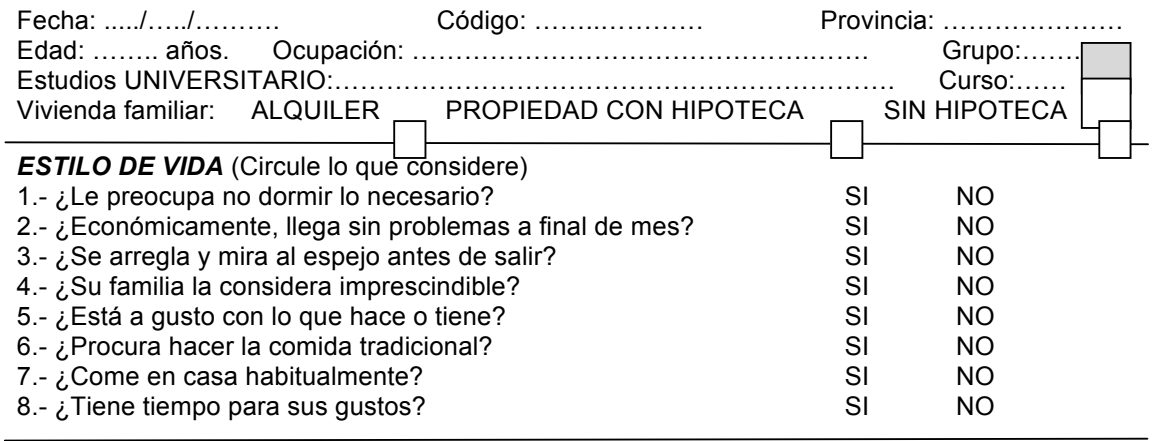

\section{ESTADO DE SALUD (Circule lo que considere)}

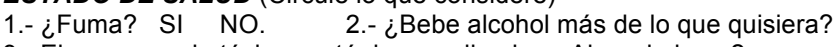

3.- El consumo de tóxicos está despenalizado. ¿Ahora lo hace?

SI $(g r . . .$.$) NO.$

4.- ¿Está tomando algún fármaco o hace algún seguimiento por

problemas de salud mental?

5.- ¿Tiene problemas de hormonas del tiroides, suprarrenales o hipófisis?

6.- ¿Ha perdido más del $10 \%$ de su peso en los últimos 6 meses?

7.- ¿Sufre alguna enfermedad?

SI NO.

En caso afirmativo indique cuál o cuáles:

SI NO.

SI NO.

SI NO.

SI NO. 
